J3eA - Vol. 2 - 10 (2003).

DOI : $10.1051 /$ bib-j3ea:2003010

\title{
Développement d'une plateforme matérielle de démonstration dédiée aux turbo codes
}

\author{
Ch. Jégo **, A. Picart ${ }^{* *}$ et J. Tousch ${ }^{* * *}$ (ENST Bretagne) \\ Mis en ligne le 15 juillet 2003.
}

\begin{abstract}
Résumé
SOPC, acronyme pour System On Programmable Chip, peut se traduire par système sur puce programmable. Cette technologie correspond à l'intégration des ressources logicielles et matérielles sur une même puce programmable de type FPGA. Depuis peu, la société Altera (fabricant de circuits reconfigurables) propose un environnement de conception de SOPC. Une étude sur cette technologie innovante, à travers un projet de formation, a été menée au sein de l'ENST Bretagne. L'objectif de ce projet était le développement d'une plateforme de démonstration pour les turbo codes à partir de l'environnement de prototypage SOPC d'Altera. Cette plateforme permet la saisie d'une image à partir d'une webcam, son traitement avant émission dans une première carte et son traitement à la réception à l'aide d'une seconde carte. Un canal de transmission a également été modélisé à partir d'un générateur de bruit blanc gaussien puis intégré sur la première carte.
\end{abstract}

Mots-clés : projet d'ingénieur, communications numériques, turbo codes en bloc, SOPC (system On Programmable Chip).

(C) EDP Sciences, 2003.

Niveau de connaissances requis. Notions de communications numériques et de conception de circuit. Niveau des étudiants. Deuxième année d'école d'ingénieur, deuxième cycle universitaire (maîtrise EEA, GEII, $\mathrm{Bac}+4)$.

* Christophe Jégo ${ }^{1}$ est Maître de Conférences au département Électronique de l'École Nationale Supérieure des Télécommunications de Bretagne (ENST Bretagne). Il est membre du groupe « algorithmes et circuits pour les communications » du laboratoire de Traitement Algorithmique et Matériel de la Communication, de l'Information et de la Connaissance (TAMCIC), unité FRE associée CNRS. Ses travaux de recherche portent sur l'application des méthodologies et des modèles pour la conception et l'exploration des systèmes sur puce au domaine des Turbo Communications.

a e-mail : christophe.jego@enst-bretagne.fr (auteur de correspondance)

** Annie Picart ${ }^{2}$ est Ingénieur d'Études au département Signal \& Communications de l'École Nationale Supérieure des Télécommunications de Bretagne (ENST Bretagne). Docteur en traitement du signal et télécommunications, elle est membre du groupe « algorithmes et circuits pour les communications »du laboratoire de Traitement Algorithmique et Matériel de la Communication, de l'Information et de la Connaissance (TAMCIC), unité FRE associée CNRS. Ses travaux de recherche portent sur les transmissions numériques, le codage canal et les techniques itératives de traitement de l'information.

e-mail : annie.picart@enst-bretagne.fr

*** Jacky Tousch ${ }^{3}$ est diplômé de l'École Nationale Supérieure des Télécommunications de Bretagne (ENST Bretagne). Il est cofondateur de la société TurboConcept. Créée en octobre 1999 à Brest, TurboConcept développe et commercialise des circuits intégrés pour les communications numériques. Expert en codage 
correcteur d'erreurs, spécialiste des turbo codes et plus généralement des techniques itératives, TurboConcept propose une gamme d'IP cores flexibles et performants.

e-mail : jacky.tousch@turboconcept.com

${ }^{1}$ Département Électronique, Technopôle Brest-Iroise, CS 83818, F-29238 Brest Cedex, France.

${ }^{2}$ Département Signal \& Communications, Technopôle Brest-Iroise, CS 83818, F-29238 Brest Cedex, France.

${ }^{3}$ TurboConcept, Technopôle Brest-Iroise, 115 rue Claude Chappe, F-29280 Plouzané, France.

\section{Introduction}

Au cours d'un projet qui a eu lieu dans le cadre de la formation d'ingénieur, six étudiants de l'ENST Bretagne ont travaillé sur l'intégration d'une chaîne de transmission numérique à base de turbo code. Les turbo codes sont un nouveau type de code correcteur d'erreurs, introduits avec un algorithme de décodage pratique en 1993 par une équipe de chercheurs de l'ENST Bretagne [1]. Ils constituent aujourd'hui la technique de codage de canal la plus performante pour la protection de l'information. Le partenaire industriel de ce projet, la société TurboConcept, est une jeune société développant des circuits utilisés dans des systèmes de transmission numérique. Cette société fondée en 1999 a pour vocation de proposer des modules de codage correcteurs d'erreurs, en particulier des IP Cores turbo codes. L'intérêt de ce projet réside dans le développement et la mise en place d'un environnement de démonstration dédié aux turbo codes pour la société TurboConcept mais également pour les départements Électronique et Signal \& Communications de l'ENST Bretagne. Ainsi, cette plateforme matérielle doit permettre de visualiser l'efficacité d'un turbo code pour corriger les erreurs de transmission et améliorer la qualité de la communication sur une image capturée en temps réel.

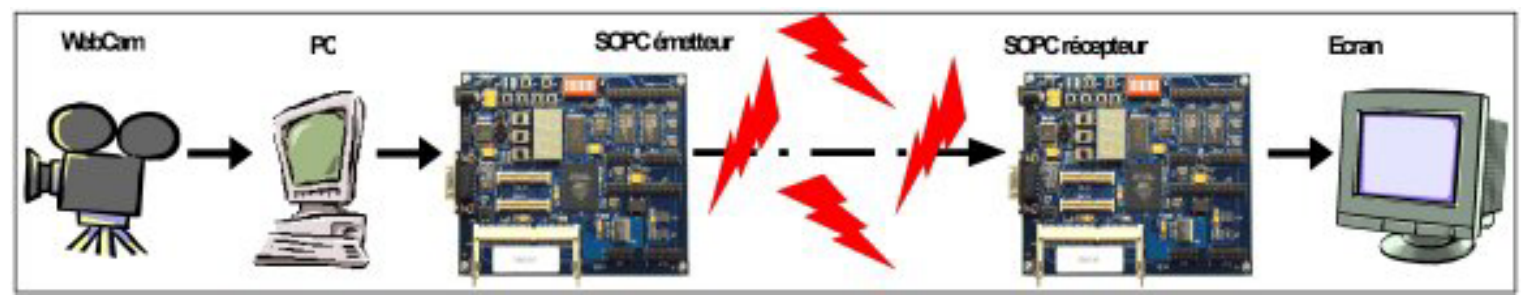

Fig. 1. Environnement de démonstration matériel dédié aux turbo codes.

Tout d'abord, nous présentons dans cet article le projet d'ingénieur qui a lieu au cours de la formation d'ingénieur de l'ENST Bretagne et l'environnement de conception qui a été utilisé par les élèves. Puis, nous détaillons la chaîne de transmission numérique qui a été développée. Enfin, le traitement d'une image numérique par la plateforme dédiée aux turbo codes est explicité.

\section{Le projet d'ingénieur dans la formation ingénieur de l'ENST Bretagne}

La formation par le projet est l'un des axes majeurs de la pédagogie de l'ENST Bretagne [2]. Ainsi, les élèves ingénieurs sont amenés à effectuer au cours des trois années différents projets (projet d'algorithmique et de programmation, projet expérimental, projet ingénieur et projet d'entreprendre). L'objectif est de confronter les élèves à des problèmes concrets pour favoriser l'apprentissage et l'expérimentation de nouvelles connaissances (langages, méthodes, outils...).

\subsection{Présentation du projet d'ingénieur}

$\mathrm{Au}$ cours de la deuxième année de la formation d'ingénieur, les élèves de l'ENST Bretagne doivent réaliser un véritable projet d'ingénieur proposé par des enseignants-chercheurs en partenariat avec un industriel. En effet, outre le suivi au quotidien de l'avancement du projet, le groupe d'enseignants a en charge la recherche et la négociation des sujets de projets avec les partenaires industriels. Ce projet d'ingénieur est un élément important 
du projet formation de l'ENST Bretagne qui, démarré en 1995, a abouti à une remise en forme complète du cursus de formation des ingénieurs télécom. D'une durée de 120 heures par étudiant, ce projet vise à la réalisation par un groupe de six à huit personnes, d'un produit télécom qui est présenté lors d'un forum réunissant industriels et partenaires institutionnels. Vitrine du savoir-faire technique des étudiants de l'école, ce projet est également l'occasion de se familiariser avec le travail de groupe et les techniques de gestion de projet. De ce fait, les trois objectifs principaux du projet ingénieur sont :

- la mise en pratique des techniques de gestion de projet en s'organisant autour d'un groupe pluridisciplinaire associant étudiants et enseignants.

- la possibilité pour les élèves d'utiliser dans un projet les connaissances théoriques et pratiques acquises dans le tronc commun du cursus de formation. De plus, la réalisation du projet est l'occasion de confronter l'élève ingénieur à la nécessité d'acquérir de nouveaux savoirs et savoir-faire et de mieux maîtriser les problèmes que pose le travail en équipe. Ceci permet aux élèves de faire un bilan global de leurs connaissances, facilitant ainsi leurs choix de formation pour les deux derniers semestres.

- la validation de la formation au travers d'activités et de missions proches de celles que les élèves auront à assumer lors de leur premier emploi, dans le cadre de la mise en oeuvre d'un projet ou d'un service de télécom.

Les projets sont encadrés par au moins deux enseignants-chercheurs pour la partie technique et par un enseignant-chercheur différent dans le cadre de la gestion de projet.

\subsection{Déroulement du projet d'ingénieur}

Les sujets sont présentés aux étudiants au cours du premier semestre de la deuxième année de formation. Les sujets doivent être liés au secteur de l'électronique, de l'informatique, du traitement de signal et plus généralement au secteur des technologies de l'information et de la communication ou à celui des services. Les élèves ont la possibilité de consulter de manière approfondie les sujets sur le site web à l'URL : http://projetingenieur.enst-bretagne.fr/.

Le projet proprement dit se déroule lors du second semestre de la seconde année de formation [3] et s'organise en trois phases :

- une phase préparatoire (de janvier à février) pendant laquelle les élèves appréhendent le contexte et effectuent la bibliographie sur le sujet. Durant cette période, les premiers contacts industriels-élèves sont pris. À l'issue de cette phase, un plan de développement est fourni par les élèves (Fig. 2).

- une phase de développement (mars à juin) pendant laquelle le partenaire industriel et les responsables enseignants du projet sont tenus au courant de l'avancement des travaux et de la tenue des délais au travers de compte-rendus d'avancement.

- une phase de mise en valeur du travail au travers d'une soutenance devant un jury et d'une journée de promotion lors d'un forum. 


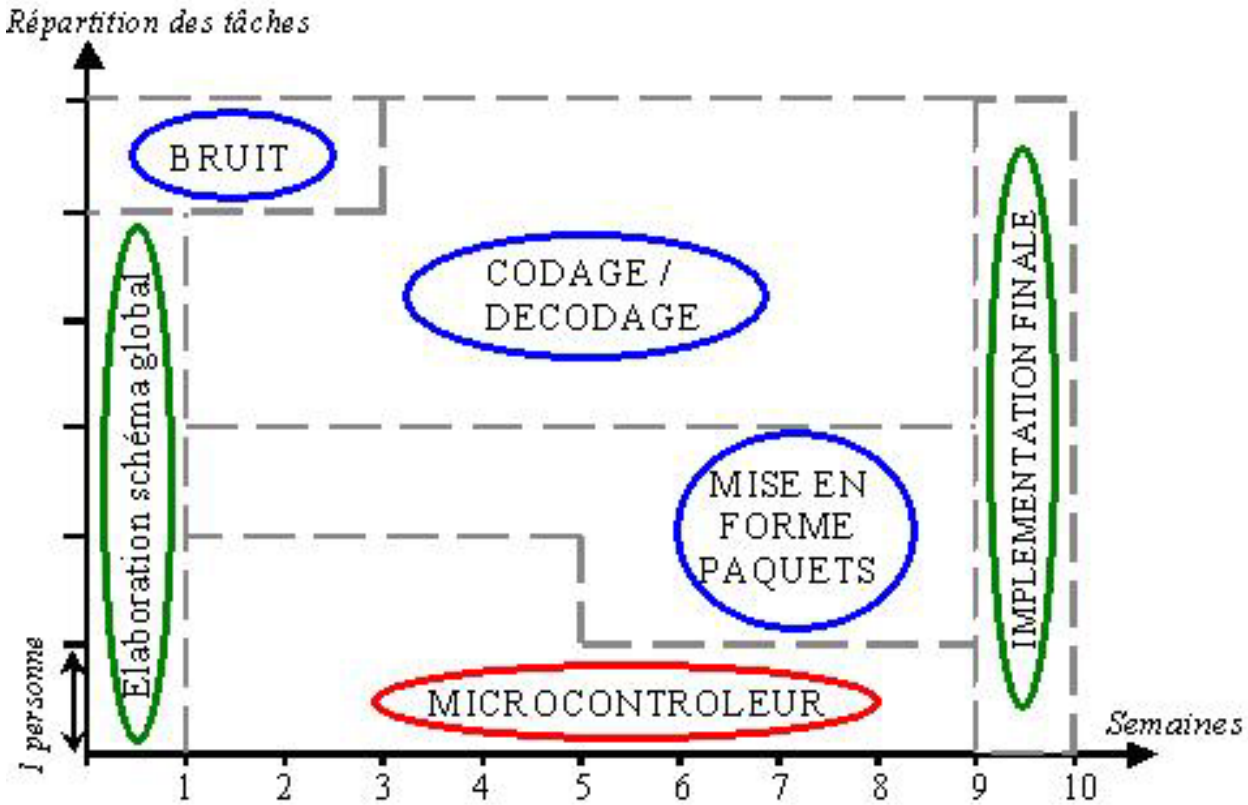

Fig. 2. Plan de développement proposé par les élèves.

\section{Environnement de prototypage utilisé lors du projet}

L'environnement de conception qui a été utilisé dans le cadre de ce projet est l'ensemble de prototypage « System on Chip » Excalibur de la société Altera. Il comprend deux cartes de développement (composées chacune d'un APEXTM 20K200E, de mémoires FLASH et RAM et de connecteurs d'extension), un processeur embarqué Nios $^{\mathrm{TM}}[4,5]$ incluant un CPU RISC sous forme d'IP, un logiciel GnuPro ${ }^{\mathrm{TM}}$ de la société Cygnus ${ }^{\mathrm{TM}}$ et un logiciel Quartus II de la société Altera. L'achat de cet ensemble a été réalisé dans le cadre du programme universitaire CNFM-Altera. Les éléments constituant l'environnement de conception sont présentés sur la figure 3.

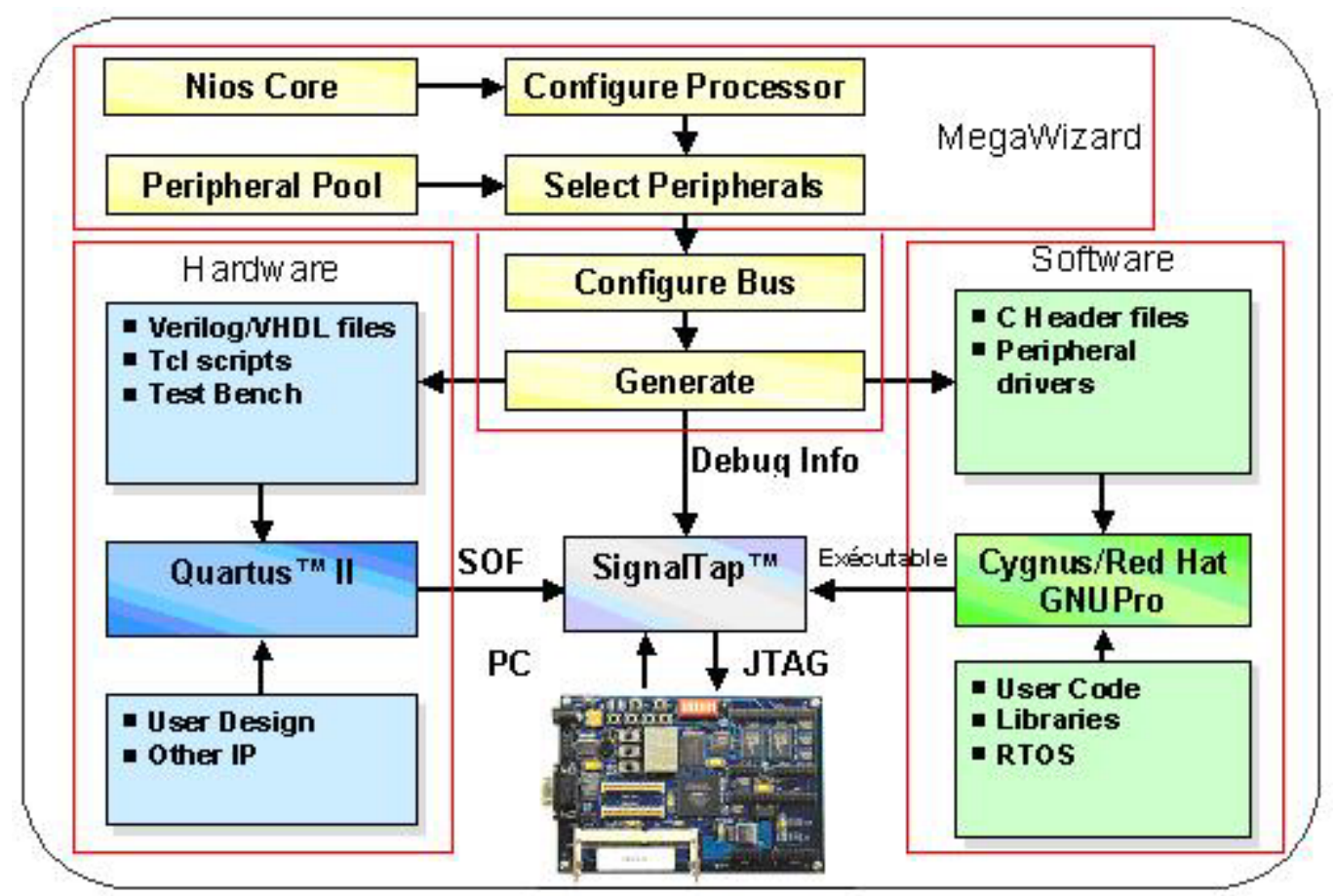

Fig. 3. Environnement de conception pour le projet. 
Cet environnement dédié à la conception de système sur puce programmable (SOPC) comprend trois parties distinctes. Tout d'abord, l'assistant MegaWizard permet de personnaliser les paramètres du coeur de processeur et de choisir le nombre et le type de périphériques associés, y compris la mémoire. Une fois le processeur paramétré, une description VHDL synthétisable est générée de manière automatique, puis ajoutée au système à concevoir. L'intégration sur FPGA des blocs matériels s'effectue à partir de l'environnement Quartus II. Dans cet environnement, le processeur est modélisé par une « boîte noire » à laquelle se connectent les différents blocs matériels du système. Un simulateur VHDL Modelsim est utilisé pour la validation des étapes de synthèse. Les résultats de synthèse des différents blocs matériels sont présentés dans le paragraphe 4. Enfin, les outils compilateur/linker/debugger C-C++ de la chaîne de développement Cygwin GnuPro ont été utilisés pour la spécification des tâches logicielles. Cette chaîne de développement permet l'écriture et la compilation des programmes devant être exécutés par un processeur.

L'environnement de conception se compose, comme nous venons de le voir, de différents outils de modélisation, de simulation et d'intégration. La plupart de ces outils sont inconnus pour les élèves de deuxième année de l'ENST Bretagne. En effet, la conception de circuits est une des options proposées en troisième année de l'école. C'est pourquoi, durant la phase préparatoire du projet (huit séances de trois heures), les élèves ont suivi plusieurs tutoriaux pour faciliter la prise en main de l'environnement de conception de SOPC. Les séances de tutorat concernaient l'apprentissage du langage VHDL, la prise en main de l'outil Quartus II, la spécification de processeurs sous MegaWizard et le développement d'applications sous Cygwin GnuPro.

\section{La chaîne de transmission dédiée aux turbo codes}

Dans le cadre de ce projet, les élèves ont développé une chaîne de transmission numérique contenant un procédé de codage/décodage canal (turbo code) et une modélisation d'un canal gaussien (Fig. 4).

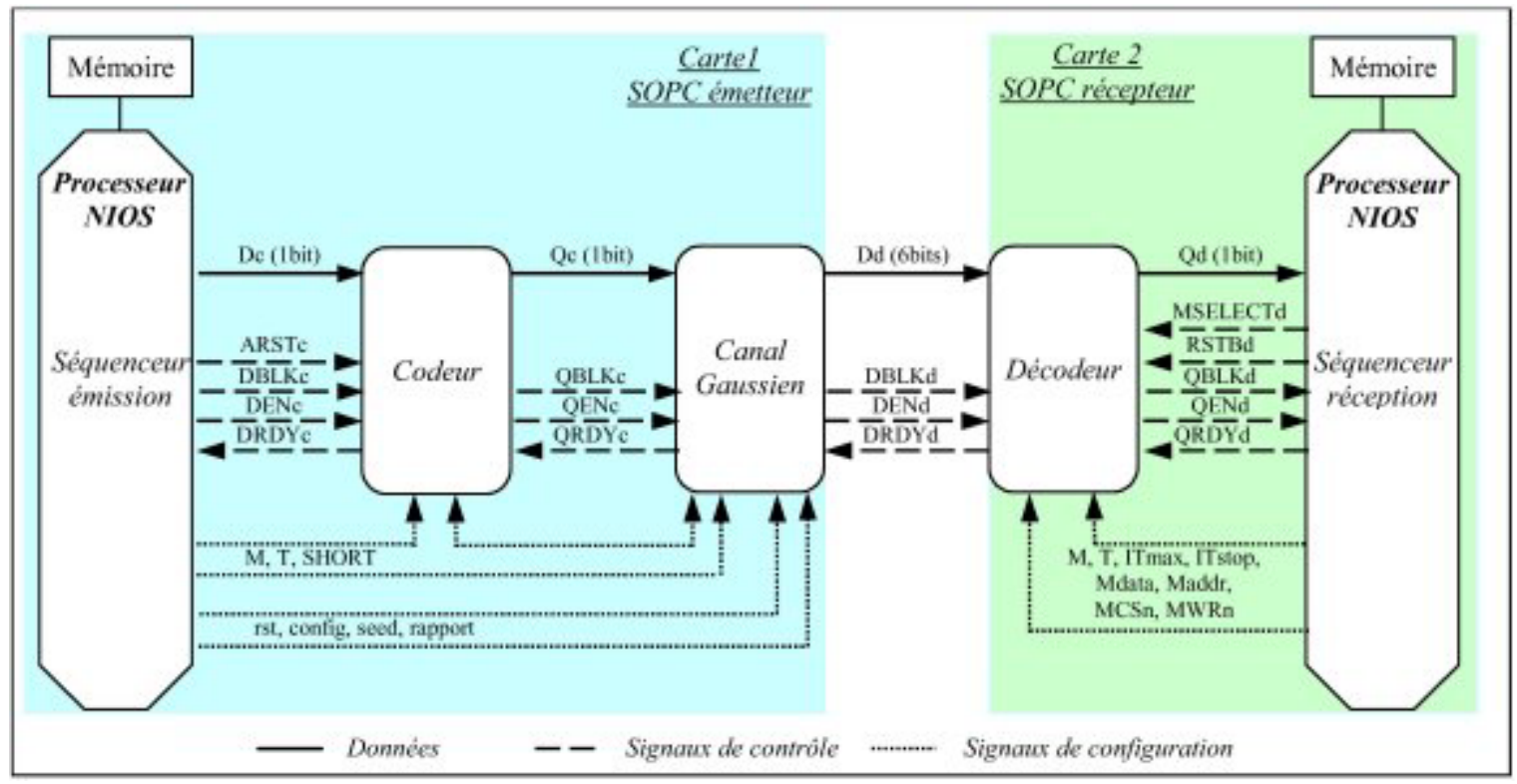

Fig. 4. Schéma de la chaîne de transmission numérique.

Cette chaîne se compose de deux cartes de développement. Elles correspondent respectivement aux SOPCs émetteur et récepteur. Le SOPC émetteur est constitué d'un IP processeur Nios, d'un IP codeur TC3000 et d'un IP modèle de canal gaussien. Le SOPC récepteur est quant à lui composé d'un IP processeur Nios et d'un IP décodeur TC3000. Un IP (Intellectual Property) se définit comme un ensemble de fichiers (modèle abstrait pour une évaluation au niveau système, description synthétisable, banc de test...) permettant l'intégration d'un bloc fonctionnel préconçu dans le flot de conception d'un système à implanter sur ASIC ou FPGA [6]. Le format ainsi que les degrés de description d'un IP font l'objet de recommandations et de standards [7]. Dans la suite de ce paragraphe, nous présenterons les différents IPs qui ont été utilisés au cours du projet. Trois différents types de signaux facilitent la gestion et la transmission des informations entre les différents modules et/ou les deux cartes. Tout d'abord les signaux de configuration sont envoyés aux blocs codage, canal et décodage. Ils permettent la définition de différents paramètres comme la taille des blocs échangés, le rapport signal à bruit, le nombre 
d'itérations de décodage... Les signaux de contrôle quant à eux garantissent les transferts de données entre les éléments ainsi que les cadencements. Enfin, les informations binaires sont échangées par l'intermédiaire de bus de données.

\subsection{Codage/décodage}

L'évolution des télécommunications s'accompagne d'une demande de qualité de transmission toujours plus importante. Une des solutions consiste alors à utiliser des codes correcteurs d'erreurs (codage canal) pour augmenter les performances de transmission. Le principe de ces codes consiste à introduire de la redondance dans le message numérique pour le protéger contre les erreurs de transmission. Deux grandes classes de code correcteur d'erreurs existent : les codes convolutifs et les codes en blocs. Les turbo codes sont des codes correcteurs d'erreurs obtenus à partir d'une concaténation de plusieurs codes classiques (convolutifs ou en blocs). Les turbo codes sont une illustration du principe selon lequel un traitement complexe peut, sous certaines conditions, être remplacé par des traitements élémentaires répétés de manière itérative. Le principe de base est d'utiliser deux codeurs élémentaires de faible complexité, séparés par une fonction d'entrelacement introduisant de la diversité. La séquence de données est codée deux fois, mais dans des ordres différents, et produit deux séquences de redondance. Deux décodeurs élémentaires sont implémentés en réception, l'un travaillant sur les données dans l'ordre naturel, l'autre sur les données permutées. Chaque décodeur élémentaire produit une information de fiabilité, appelée information extrinsèque, qui est utilisée par l'autre décodeur pour améliorer ses performances. Ce processus itératif est le turbo décodage. Au niveau performance, un turbo code apporte généralement un gain de 2 à $4 \mathrm{~dB}$ par rapport aux solutions de codage correcteur d'erreurs classiques.

C'est en 1993 que les turbo codes, inventés dans les départements Électronique et Signal \& Communications de l'ENST Bretagne (Berrou, Glavieux), ont été présentés à la communauté scientifique internationale [1]. Ils ont suscité un intérêt considérable, autant dans le monde académique (plus de 4000 publications recensées à ce jour sur le sujet) que dans le monde industriel et dans les comités de normalisation (CCSDS, UMTS, DVB-RCS, ...). En 1994, des chercheurs de l'ENST Bretagne [8] ont proposé une méthode de décodage basée sur le principe de turbo décodage [1] et à partir de l'algorithme de Chase. Cette méthode, appelée turbo décodage de codes produits, donne de bonnes performances en terme de taux d'erreur binaire au bout de 4 à 5 itérations pour une complexité matérielle relativement faible.

TC3000 est une famille de turbo codes de codes produits développée par la société TurboConcept. Les coeurs d'IP codeur [9,10] et décodeur [11,12] de cette famille permettent l'implémentation de codes de parité (détecteur d'erreur), de Hamming (de pouvoir de correction $t=1$ ) ou $\mathrm{BCH}$ (de pouvoir de correction $t=2$ ). Trois niveaux de débit binaire compris entre 7 et $25 \mathrm{Mbits} / \mathrm{s}$ peuvent être choisis. La figure 5 présente la complexité matérielle (nombre de cellule logique) des différentes solutions architecturales du décodeur et le débit binaire correspondant. Naturellement, la complexité matérielle dépend du type de code sélectionné : parité, Hamming et/ou BCH. De plus, le débit binaire est directement fonction de la famille de FPGA. 


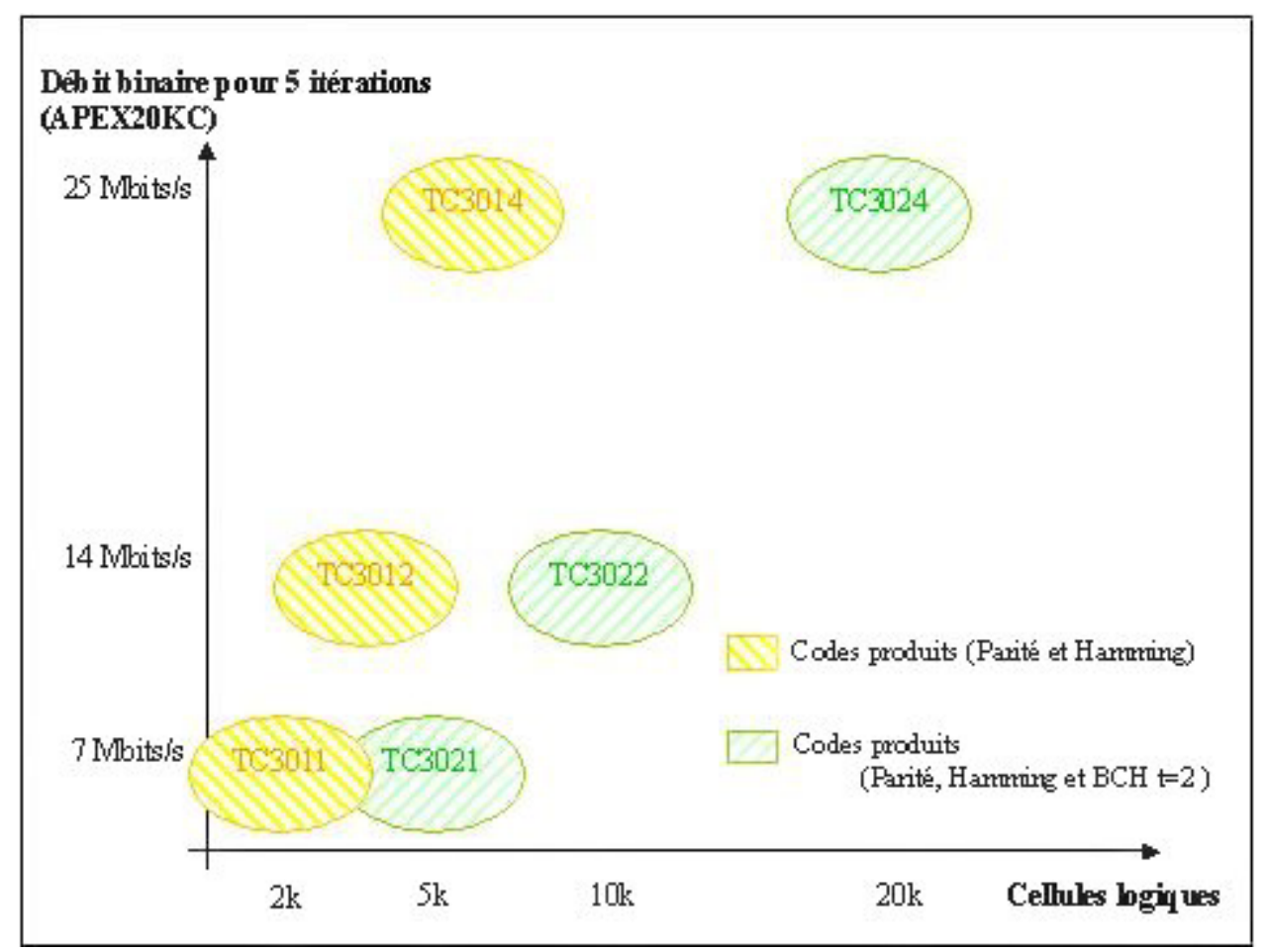

Fig. 5. Débit binaire versus complexité matérielle.

Au cours de ce projet, des IPs codeur et décodeur ont été synthétisés. Ils exécutent des codes de parité et de Hamming sur des blocs allant de 16 à 4096 bits. Les résultats de synthèse pour un code de Hamming étendu $(64,57)$ de rendement 0,89 sont détaillés dans le tableau 1 .

\begin{tabular}{|c|c|c|c|c|c|}
\hline & $\begin{array}{c}\text { Nombre de } \\
\text { cellules logiques }\end{array}$ & $\begin{array}{c}\text { Nombre de } \\
\text { cellules mémoires }\end{array}$ & $\begin{array}{c}\text { Fréquence } \\
\text { d'horloge } \\
\text { max. }\end{array}$ & $\begin{array}{c}\text { Débit d'entrée } \\
\text { Hamming (64,57) }\end{array}$ & $\begin{array}{c}\text { Débit de sortie } \\
\text { Hamming (64,57) }\end{array}$ \\
\hline Codeur & $\begin{array}{c}178 / 8320 \\
(2 \%)\end{array}$ & $\begin{array}{c}1 / 52 \\
(2 \%)\end{array}$ & $100 \mathrm{MHz}$ & $79 \mathrm{Mbits} / \mathrm{s}$ & $100 \mathrm{Mbits} / \mathrm{s}$ \\
\hline Décodeur & $\begin{array}{c}2631 / 8320 \\
(31 \%)\end{array}$ & $\begin{array}{c}30 / 52 \\
(57 \%)\end{array}$ & $43 \mathrm{MHz}$ & $\begin{array}{c}3,88 \mathrm{Mbits} / \mathrm{s} \\
(\text { bloc } 4096 \mathrm{bits})\end{array}$ & $\begin{array}{c}3,05 \mathrm{Mbits} / \mathrm{s} \\
(\mathrm{bloc} 3246 \mathrm{bits})\end{array}$ \\
\hline
\end{tabular}

Tab. 1. Résultats de synthèse du codeur et du décodeur pour cinq itérations ciblant un APEX20K200EFC484-2x.

L'occupation en cellules logiques et en cellules mémoires représente à peine $2 \%$ des capacités de la cible FPGA retenue pour le codeur. Par contre, la complexité nécessaire à l'implémentation du décodeur pour cinq itérations est bien plus importante. En effet, $31 \%$ des cellules logiques et $57 \%$ des cellules mémoires sont occupées. C'est principalement pour cette raison que la modélisation du canal gaussien est implémentée sur la carte émettrice plutôt que sur la carte réceptrice. Au niveau des débits, le débit atteint en sortie du décodeur est de $3 \mathrm{Mbits} / \mathrm{s}$. Des débits plus importants peuvent être atteints mais ces performances ont un coût matériel.

\subsection{Canal gaussien}

La chaîne de communication numérique nécessite la modélisation d'un canal de transmission. Dans notre cas, nous avons choisi de simuler un canal gaussien à partir d'un générateur matériel de bruit blanc gaussien (GBBG). La spécification d'un bruit blanc gaussien correspond à la génération d'une variable aléatoire devant suivre la loi

$$
\sigma^{2}=\left(\frac{2 E}{N_{0}}\right)^{-1}
$$

gaussienne (moyenne nulle, variance

avec $\mathrm{E} / \mathrm{N}_{0}$ rapport signal à bruit). L'IP générateur de bruit blanc gaussien que nous utilisons a été développé par le laboratoire LESTER de l'Université de Bretagne Sud 
[13]. Ce GBBG se caractérise par une grande précision (densité de probabilité dite conforme à $(4 \sigma, 1 \%)$ ) et un faible coût matériel. Il utilise conjointement la méthode de Box-Muller et le théorème de la limite centrale [14]. La figure 6 présente l'architecture du canal gaussien comprenant l'IP GBBG. Le bloc LFSR (Linear Feedback Shift Register) contient des registres à décalage avec rétroaction linéaire qui servent à générer les variables aléatoires binaires pseudo-aléatoires. La méthode de Box-Muller consiste à générer une variable aléatoire $x(0,1)$ à partir de deux variables aléatoires réelles indépendantes $x_{1}$ et $x_{2}$ uniformément distribuées sur l'intervalle $[0,1]$ telle que :

$$
x=f\left(x_{1}\right) \cdot g\left(x_{2}\right) \text { avec } f\left(x_{1}\right)=\sqrt{-\ln \left(x_{1}\right)} \text { et } g\left(x_{2}\right)=\sqrt{2} \cos \left(2 \pi x_{2}\right)
$$

Le bloc LFSR permet la génération des variables $x_{1}$ et $x_{2}$. Les calculs de $f\left(x_{1}\right)$ et de $g\left(x_{2}\right)$ sont effectués par des tables implémentées dans des ROMs. Enfin, l'accumulateur de quatre valeurs successives est utilisé pour l'application du théorème de la limite centrale. L'ensemble de ces ressources constitue l'IP GBBG. Les variables aléatoires obtenues ont un écart type de 1, c'est pourquoi la modélisation d'un canal gaussien nécessite la multiplication des variables générées par la valeur de l'écart type désiré. En fait, cette valeur d'écart type est

$$
\sigma^{2}=\left(\frac{2 E}{N_{\sigma}}\right)^{-1}
$$

directement fonction du rapport signal à bruit fixé ( $\quad$ ). La ROM de génération de l'écart type à partir du rapport signal à bruit qui a été implémentée au cours de cette étude permet la simulation d'un canal gaussien pour des rapports signal à bruit variant de $-2 \mathrm{~dB}$ à $13,75 \mathrm{~dB}$ par pas de $0,25 \mathrm{~dB}$.

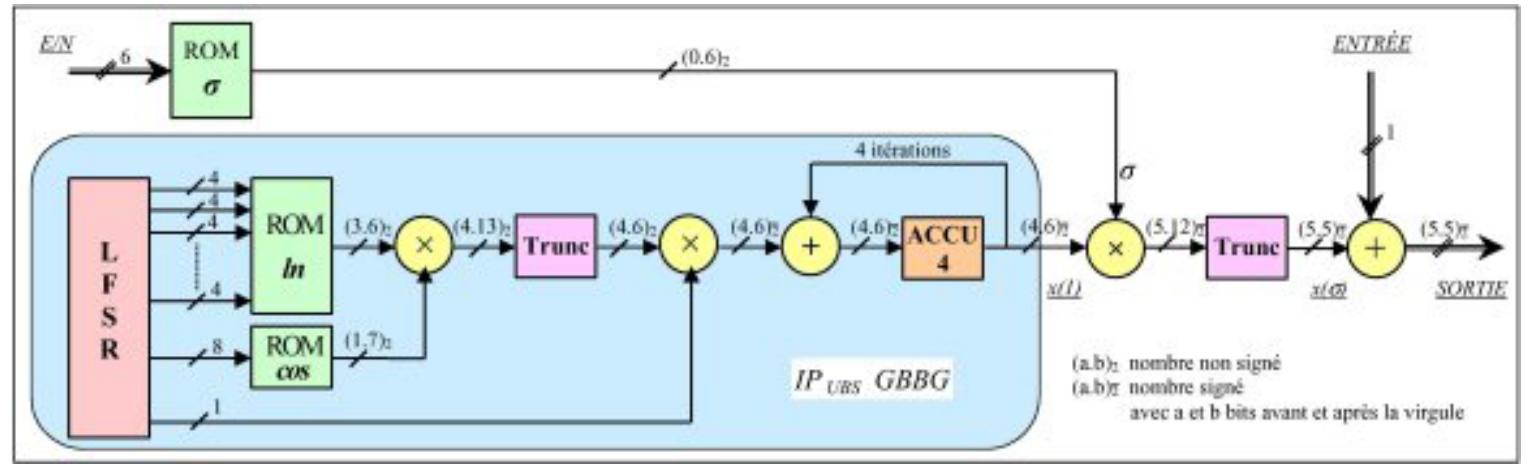

Fig. 6. Architecture du canal gaussien.

Le tableau 2 donne les caractéristiques matérielles du canal gaussien obtenu. La complexité totale du canal gaussien est faible au niveau du nombre de cellule logique $(7 \%)$ et tout à fait acceptable en nombre de cellule

\begin{tabular}{|c|c|c|c|c|c|}
\hline $\begin{array}{c}\text { Famille FPGA } \\
\text { d'Altera }\end{array}$ & $\begin{array}{l}\text { Plage de rapport } \\
\text { signal/bruit } \\
\text { (dB) }\end{array}$ & $\begin{array}{c}\text { Nombre de } \\
\text { cellules } \\
\text { logiques }\end{array}$ & $\begin{array}{c}\text { Nombre de } \\
\text { cellules } \\
\text { mémoires }\end{array}$ & $\begin{array}{c}\text { Fréquence } \\
\text { d'horloge } \\
\text { max. }\end{array}$ & $\begin{array}{l}\text { Débit E/S } \\
\max \end{array}$ \\
\hline $\begin{array}{c}\text { APEX20K200EFC484- } \\
2 \mathrm{x}\end{array}$ & $\begin{array}{l}{[-2 ; 13,75]} \\
\text { pas } 0,25\end{array}$ & $\begin{array}{l}592 / 8320 \\
(7 \%)\end{array}$ & $\begin{array}{l}7 / 52 \\
(13 \%)\end{array}$ & $40 \mathrm{MHz}$ & $10 \mathrm{Mbits} / \mathrm{s}$ \\
\hline
\end{tabular}
mémoire (13\%). À noter que quatre cycles d'horloge sont nécessaires pour effectuer la génération d'une variable aléatoire.

Tab. 2. Résultats de synthèse pour le canal gaussien.

À l'issue de la synthèse et de l'implémentation sur FPGA, une simulation des performances du canal gaussien a été réalisée. Les densités de probabilité de la variable aléatoire $x(\sigma)$ pour $\sigma=1,0,5$ et 0,25 qui correspondent à des rapports signal à bruit respectifs de $-3 \mathrm{~dB}, 3 \mathrm{~dB}$ et $6 \mathrm{~dB}$ sont présentées sur la figure 7 . 


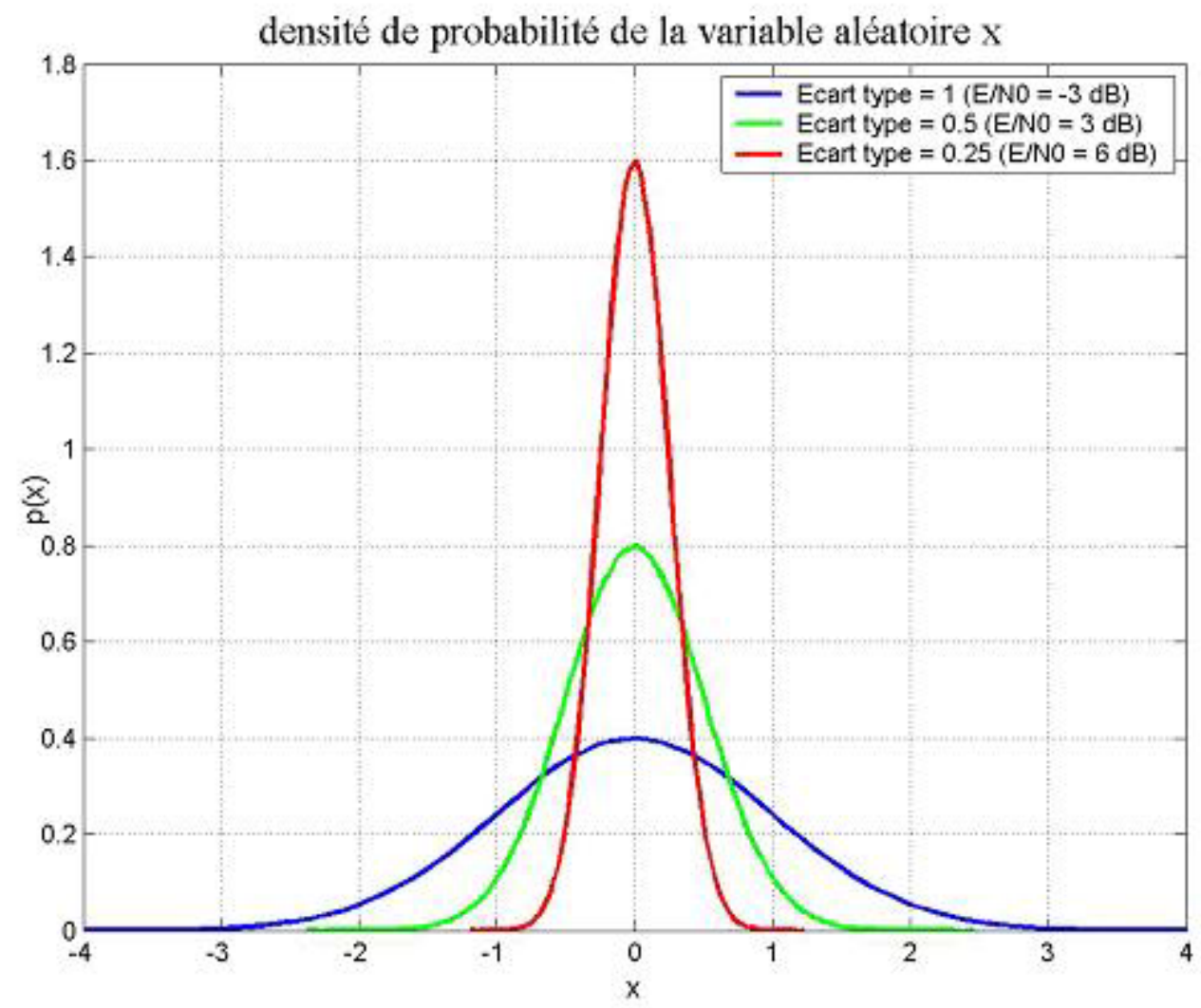

Fig. 7. Résultats de simulation du bruit gaussien sur $5 \cdot 10^{7}$ échantillons.

\subsection{Définition des séquenceurs}

L'échange des données, la gestion des signaux de contrôle et la gestion des signaux de configuration sont assurés par des processeurs paramétrables Nios développés par Altera. Le coeur de ce processeur est basé sur une architecture RISC 16 ou 32 bits [4]. Son interface bus (PBM) permet l'utilisation de plusieurs types de mémoires (interne ou externe au FPGA) et le raccordement de périphériques standards (UART, Timer, Parallel I/O). De plus, l'intégration d'un ROM_monitor associé à une liaison série RS-232 facilite la communication avec le processeur (chargement des programmes). La gestion des signaux est ainsi spécifiée dans des programmes en $\mathrm{C}$ qui s'exécutent sur les processeurs. Les configurations des processeurs Nios dédiés au contrôle des parties émission et réception de la chaîne de transmission numérique sont détaillées dans le tableau 3.

\begin{tabular}{|c|c|c|c|c|c|c|c|}
\hline & \multirow{2}{*}{$\begin{array}{l}\text { Nombre de } \\
\text { cellules } \\
\text { logiques }\end{array}$} & \multirow{2}{*}{$\begin{array}{l}\text { Nombre de } \\
\text { cellules } \\
\text { mémoires }\end{array}$} & \multirow{2}{*}{$\begin{array}{c}\text { Fréquence } \\
\text { d'horloge } \\
\text { max. }\end{array}$} & \multirow[t]{2}{*}{$\begin{array}{l}\text { Architecture } \\
\text { RISC } 32 \text { bits }\end{array}$} & \multirow[t]{2}{*}{$\begin{array}{l}\text { Mémoire interne } \\
\text { ROM_monitor }\end{array}$} & \multicolumn{2}{|c|}{$\begin{array}{l}\text { Mémoire } \\
\text { externe }\end{array}$} \\
\hline & & & & & & Flash & SRAM \\
\hline $\begin{array}{l}\text { Nios } \\
\text { émetteur }\end{array}$ & $\begin{array}{c}3115 / 8320 \\
(3 \%)\end{array}$ & $\begin{array}{l}17 / 52 \\
(32 \%)\end{array}$ & $37 \mathrm{M}$ & $256 \mathrm{res}$ & 1 Kbyte & $\begin{array}{c}1 \\
\text { Mbyte }\end{array}$ & $\begin{array}{c}256 \\
\text { Kbytes }\end{array}$ \\
\hline $\begin{array}{l}\text { Nios } \\
\text { récepteur }\end{array}$ & $\begin{array}{c}2931 / 8320 \\
(35 \%)\end{array}$ & $\begin{array}{l}17 / 52 \\
(32 \%)\end{array}$ & $37 \mathrm{MHz}$ & 256 registres & 1 Kbyte & $\begin{array}{c}1 \\
\text { Mbyte }\end{array}$ & $\begin{array}{c}256 \\
\text { Kbytes }\end{array}$ \\
\hline
\end{tabular}

Tab. 3. Résultats de synthèse des processeurs NIOS ciblant un APEX20K200EFC484-2x.

Les occupations en cellules logiques et en cellules mémoires sont similaires pour les processeurs des cartes SOPC émetteur et SPOC récepteur. Elles correspondent environ au tiers des ressources de chaque FPGA.

Globalement l'ensemble des ressources implémentées sur la carte émettrice (processeur Nios, turbo codeur et canal gaussien) occupent à peu près la moitié des cellules logiques et des cellules mémoires. Ces résultats sont intéressants car ils laissent la possibilité d'ajouter d'autres fonctionnalités sur la carte émettrice. Il est ainsi possible de remplacer le canal gaussien par d'autres canaux plus complexes comme par exemple un canal de 
rayleigh. Des travaux sur l'implémentation d'un canal de rayleigh sont actuellement en cours de développement au sein du département Électronique de l'ENST Bretagne. L'ensemble processeur et turbo décodeur occupe quant à lui $70 \%$ des cellules logiques et la quasi-totalité des cellules mémoires (88\%). Par conséquent, l'implémentation de turbo décodeurs plus rapides nécessite l'emploi de FPGA contenant plus de ressources comme par exemple le FPGA APEX20K1000.

\section{Traitement d'une image numérique}

L'objectif du projet est d'illustrer l'intérêt des turbo codes à travers la transmission numérique d'une image. Pour ce faire, il faut d'une part transférer une image acquise sur un ordinateur vers la carte 1 (SOPC émetteur) et d'autre part être capable de récupérer l'image transmise sur la carte 2 (SOPC récepteur). L'environnement défini (Fig. 8) permet de traiter des images de taille 640*480 pixels sur 256 couleurs au format bitmap. L'image initiale est convertie au format exécutable . srec puis chargée en mémoire par le processeur Nios. Au niveau de la réception, l'image qui se trouve en mémoire est lue par le processeur puis soit transformée en bitmap soit directement disponible au niveau d'une sortie VGA. L'interface VGA modélisée par Groeneveld [15], est un contrôleur vidéo qui comprend un IP Core Lancelot et une carte Lancelot (Vidéo DAC) qui se branche sur les connecteurs d'extensions de la carte de développement Excalibur d'Altera.

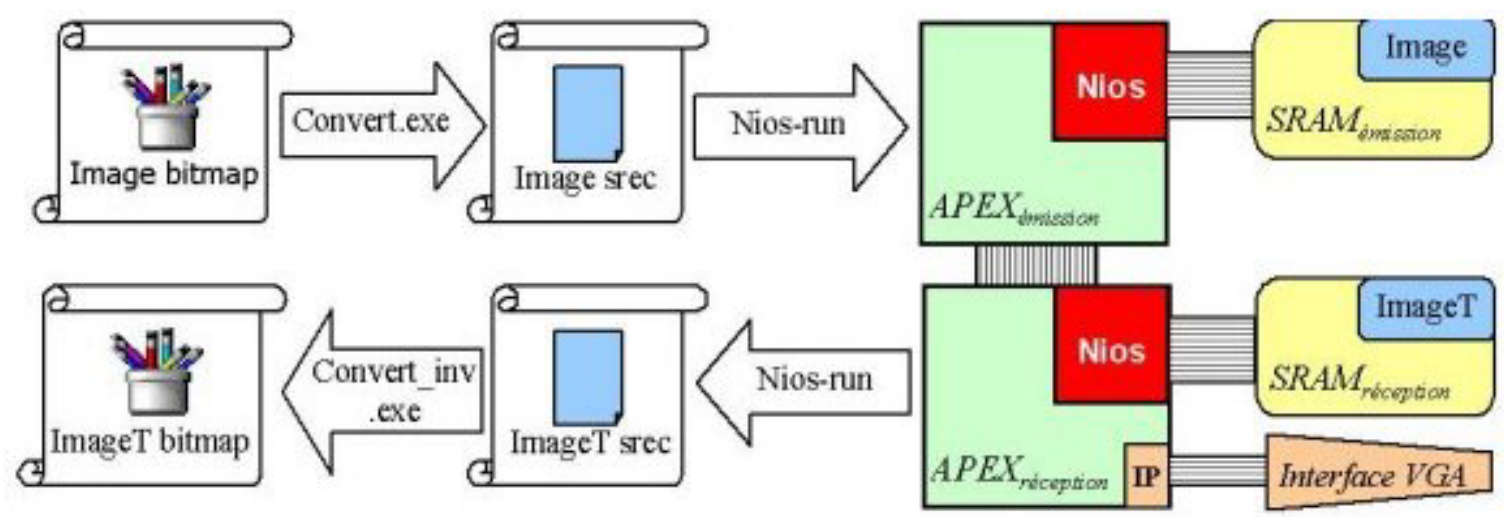

Fig. 8. Environnement de traitement pour une image numérique.

\section{Conclusion}

Un projet sur le développement d'une plateforme de démonstration pour les turbo codes à partir de l'environnement de prototypage SOPC d'Altera a été proposé à des élèves ingénieurs de l'ENST Bretagne. Ce projet fut pour les six élèves une première expérience dans le domaine de la conception de circuit numérique qui a permis d'appréhender les notions de conception matérielle et logicielle de système sur puce programmable. Ainsi, au cours de cette étude, ils se sont familiarisés avec les différents outils composant un environnement de conception système. De plus, ils ont également étudié le concept de composant virtuel (IP) et les contraintes de conception correspondantes [16]. En particulier, le travail sur les produits IP Cores turbo codes de la société TurboConcept fut une expérience formatrice.

\section{Références bibliographiques}

[1] C. Berrou, A. Glavieux et P. Thitimajshima, Near Shannon limit error correcting coding and decoding: Turbo Codes, Proceedings of the IEEE International Conference on Communication ICC93, May 1993, 2/3 (1993).

[2] Groupe de pilotage du projet ingénieur de l'ENST Bretagne, VADE-MECUM du projet d'ingénieur 20012002, (ENST Bretagne, Janvier 2002).

[3] ENST Bretagne, Pédagogie par projet dans l'enseignement supérieur : enjeux et perspectives, Actes du $1^{\mathrm{er}}$ 
colloque, Brest, France, juin 2001.

[4] Altera, NIOS Embedded Processor - Software development reference manual, version 2.1 (avril 2002).

[5] Altera, NIOS Tutorial, version 1.1 (avril 2002).

[6] M. Keating, P. Bricaud et R. J. Rickford, Reuse Methodology Manual for System-on-a-Chip Designs, 3 e éd. (Kluwer Academic Publishers, 2002).

[7] VSI alliance, Architecture Document, version 2.6 (mai 2002).

[8] R. Pyndiah, A. Glavieux, A. Picart et S. Jacq, Near optimum decoding of product codes, GLOBECOM94, novembre 1994.

[9] TurboConcept, TC3000 turbo product code encoder : Data sheet, version 1.0 (août 2001).

[10] TurboConcept, TC3000 turbo product code encoder : User guide, version 1.0 (août 2001).

[11] TurboConcept, TC3000 turbo product code decoder : Data sheet, version 1.0 (janvier 2002).

[12] TurboConcept, TC3000 turbo product code decoder : User guide, version 1.0 (janvier 2002).

[13] E. Boutillon, A. Gazel, J.L. Danger, G. Gulak et H. Laamari, Un générateur de bruit blanc gaussien sur un FPGA pour la simulation rapide de systèmes de transmissions, $14^{\mathrm{e}}$ colloque du GRETSI, Toulouse, France, septembre 2001.

[14] J.L. Danger, A. Ghazel, E. Boutillon et H. Laamari, Efficient FPGA Implementation of Gaussian Noise Generator for Communication Channel Emulation, The $7^{\text {th }}$ IEEE International Conference on Electronics, Circuits \& Systems (ICECS'2K), Kaslik, Liban (décembre 2000).

[15] M. Groeneveld, Lancelot User Manual, version 1.0 (mai 2002).

[16] X. Abiven, M. Brisbarre, G. Giraud, J. Guth, O. Muller et P. Serodes, Projet $S 4 n^{\circ} 23$ : Intégration d'une chaîne de transmission numérique à base de Turbo Code, rapport de projet S4, ENST Bretagne (juin 2002). 